\title{
O-band excited state quantum dot bilayer lasers
}

\author{
M. A. Majid, ${ }^{1, a)}$ D.T.D. Childs, ${ }^{1}$ K. Kennedy, ${ }^{1}$ R. Airey, ${ }^{1}$ R. A. Hogg, ${ }^{1}$ E. Clarke, ${ }^{2}$ P. Spencer, ${ }^{2}$ \\ and R. Murray ${ }^{2}$ \\ ${ }^{1}$ Department of Electronic \& Electrical Engineering, The University of Sheffield, Centre for Nanoscience and \\ Technology, North Campus, Broad Lane, Sheffield, S3 7HQ, United Kingdom \\ ${ }^{2}$ Physics Department, Imperial College London, The Blackett Laboratory, Prince Consort Road, London SW7 \\ $2 A Z$, United Kingdom
}

(Received 9 March 2011; accepted 8 June 2011; published online 1 August 2011)

\begin{abstract}
Bilayer InAs/GaAs quantum dot (QD) lasers operating in the excited state at wavelengths that span the O-band are demonstrated. The higher saturated gain and lower scattering time of the excited states of the ensemble of QDs offers the opportunity for fast direct-modulation lasers. We predict an increase in K-factor limited modulation bandwidth from QD lasers operating in the excited state due to a reduction in carrier transport and scattering times whilst maintaining high peak modal gain. (C) 2011 American Institute of Physics. [doi:10.1063/1.3605590]
\end{abstract}

Semiconductor diode lasers incorporating self-assembled quantum dots (QDs) on GaAs substrates accessing the International Telecommunication Union (ITU) O-band (1.26-1.36 $\mu \mathrm{m})$ have received considerable attention, to the point of their current commercialization, ${ }^{1,2}$ with recent performance milestones reached including high-speed, temperature-insensitive ${ }^{3}$ operation around room temperature (RT) at $1.3 \mu \mathrm{m}$ through the use of p-type doping. ${ }^{4}$ In contrast to quantum well lasers, the modulation dynamics of QD lasers are limited by damping, ${ }^{5}$ and in order to achieve high modulation rates, low carrier scattering times into the lasing state and high saturated modal gains are required. Possible routes to increase saturated gain include careful optimization of growth conditions to increase QD areal density ${ }^{6}$ or to increase differential gain by increasing p-doping, but this has practical limit in terms of additional loss. ${ }^{7}$ It has recently been demonstrated that operation using the excited state (ES) of the QDs exhibit much higher damping limited bandwidths as compared to ground state (GS) lasers. ${ }^{8,9}$ This enhancement was attributed to higher saturated gain (double) and lower scattering time (half) compared to the ground state. However, this is achieved at the expense of higher operating currents and shorter operating wavelengths that may be impractical for fiber-optic based optical communications applications.

Here, we demonstrate that the technologies to realize long wavelength QD GS emission can be applied to realize QD ES emission spanning the O-band. In this paper, we report on the fabrication of QD bilayer ${ }^{10,11}$ materials where ES lasing is demonstrated between $1.26 \mu \mathrm{m}$ and $1.33 \mu \mathrm{m}$ spanning the ITU O-band. This is achieved by using QD material comprising closely stacked layers of QDs, which are strain and electronically coupled, consisting of a seed layer that determines the areal density and an upper emission layer. The wavelength coverage is made possible by utilizing both GaAs and InGaAs capping of the emission layer. Measurement of multi-section lasers containing these QD bilayers allows the peak modal gain of the ES as a function of current density to be deduced, ${ }^{12}$ and this is comparable to commercially available 1.3 $\mu \mathrm{m}$ QD laser samples operating in the GS.

${ }^{\text {a)} E l e c t r o n i c ~ m a i l: ~ a . m . m o h a m m e d @ s h e f f i e l d . a c . u k . ~}$
The samples were p-i-n edge-emitting laser structures grown on $\mathrm{n}^{+} \mathrm{GaAs}(100)$ substrates by molecular beam epitaxy. The active region for the structures was located in a 500 $\mathrm{nm}$ undoped GaAs layer sandwiched between $1500 \mathrm{~nm}$ $\mathrm{Al}_{0.33} \mathrm{Ga}_{0.67} \mathrm{As}$ doped cladding layers, with a $400 \mathrm{~nm}$ p-type GaAs:Be contact layer completing the structure. The active region consisted of five GaAs-capped or $\operatorname{In}_{0.18} \mathrm{Ga}_{0.82} \mathrm{As}(4 \mathrm{~nm})$ capped QD bilayers. We refer to these samples as the "GaAscapped bilayer" or "InGaAs-capped bilayer" sample in the following. Each bilayer consists of two closely spaced InAs/ GaAs QD layers: a seed layer and an upper emission layer. Strain fields from the underlying QDs in the seed layer penetrate the thin GaAs spacer layer (10 $\mathrm{nm}$ between QD layers), providing preferential nucleation sites for QDs in the second layer. This leads to a high degree of vertical correlation between QDs in the two layers (as observed in the transmission electron microscopy (TEM) images in Fig. 1), ${ }^{13}$ such that the seed layer acts as a template for growth in the second layer, fixing the QD density in this layer over a wide range of growth conditions. This allows a greater choice of growth conditions for QDs in the upper layer, in order to extend their emission wavelength, particularly by reducing the growth temperature. For these samples, the QD density in each layer was $2.7 \times 10^{10} \mathrm{~cm}^{-2}$. The size of the seed layer QDs is similar in both samples, but for the InGaAs-capped sample, the QDs in the upper layer remain larger and more In-rich because the out-diffusion of In from the QDs during capping is suppressed due to the InGaAs capping layer. ${ }^{14}$ The QD layers are sufficiently close for efficient electronic coupling, so that emission occurs predominantly in the upper QD layer.

Fig. 1(a) shows edge-emission RT electroluminescence (EL) spectra with increasing current density $\left(70-350 \mathrm{~A} / \mathrm{cm}^{2}\right.$ ), obtained from a device fabricated from GaAs-capped bilayer sample where lasing is inhibited (device length $1 \mathrm{~mm}$ ). Emission at $1.34 \mu \mathrm{m}$ and $1.26 \mu \mathrm{m}$ from the GaAs-capped samples is attributed to emission from the GS and ES of the second layer QDs, respectively. Fig. 1(b) shows EL spectra obtained under similar conditions from the InGaAs-capped bilayer sample. There is an extension of the GS and ES emission compared to the GaAs-capped bilayer sample to $1.42 \mu \mathrm{m}$ and $1.33 \mu \mathrm{m}$, respectively, which is due to the 


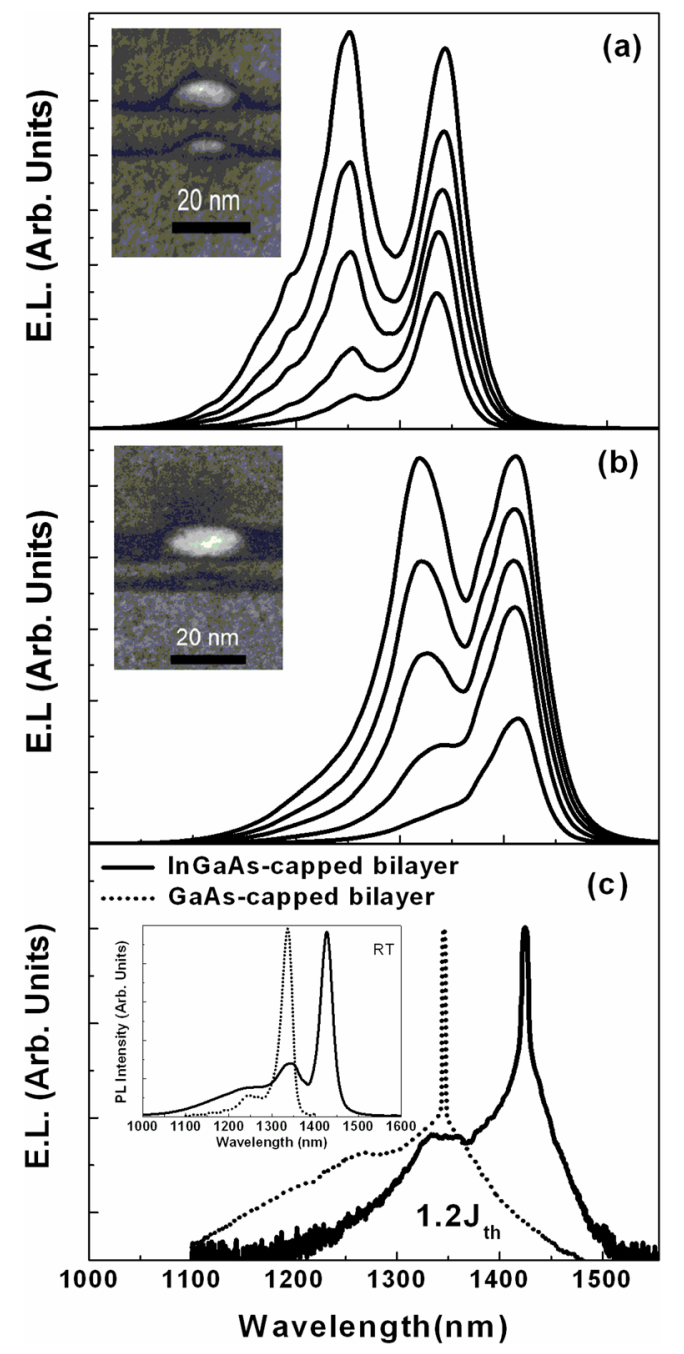

FIG. 1. (Color online) (a) Room temperature EL spectra obtained over a range of current density $\left(70-350 \mathrm{~A} / \mathrm{cm}^{2}\right)$ for GaAs-capped bilayer, Inset: Dark field (002) cross-sectional TEM images and (b) InGaAs-capped bilayer samples, Inset: Dark field (002) cross-sectional TEM images (c) Lasing spectra at $1.2 \mathrm{~J}_{\text {th }}$ obtained from $2 \mathrm{~mm}$ GaAs-capped and $8.7 \mathrm{~mm}$ InGaAscapped bilayer lasers. Inset: RT PL spectra obtained from individual GaAscapped or InGaAs-capped QD bilayers.

preservation of the QD size and composition and also the strain reduction provided by the InGaAs capping layer. RT emission from the GS of the seed layer QDs grown under these conditions is at $\sim 1.20 \mu \mathrm{m}$ and a small peak at this wavelength is only observed at high bias for GaAs-capped bilayer sample. The suppression of emission from these QDs in all samples indicates that there is an efficient electronic coupling between the QD layers in each bilayer.

Fig. 1(c) shows EL lasing spectra obtained from other devices fabricated from the GaAs-capped bilayer and the InGaAs-capped bilayer sample, respectively, at bias levels of 1.2 times the laser threshold current $\left(\mathrm{J}_{\mathrm{th}}\right)$ for each device. Both devices exhibit lasing in the ground state, at a wavelength of $1.34 \mu \mathrm{m}$ with $\mathrm{J}_{\text {th }}=83 \mathrm{~A} / \mathrm{cm}^{2}$ for the GaAs-capped bilayer and a wavelength of $1.42 \mu \mathrm{m}$ with $\mathrm{J}_{\text {th }}=550 \mathrm{~A} / \mathrm{cm}^{2}$ for the InGaAs-capped bilayer. The increased device length required to achieve GS lasing (and increased $\mathrm{J}_{\mathrm{th}}$ ) for the InGaAs-capped bilayer is a result of increased optical losses due to an increase in the roughness of one of the GaAs/ $\mathrm{AlGaAs}$ interfaces in this sample, observed by TEM (images
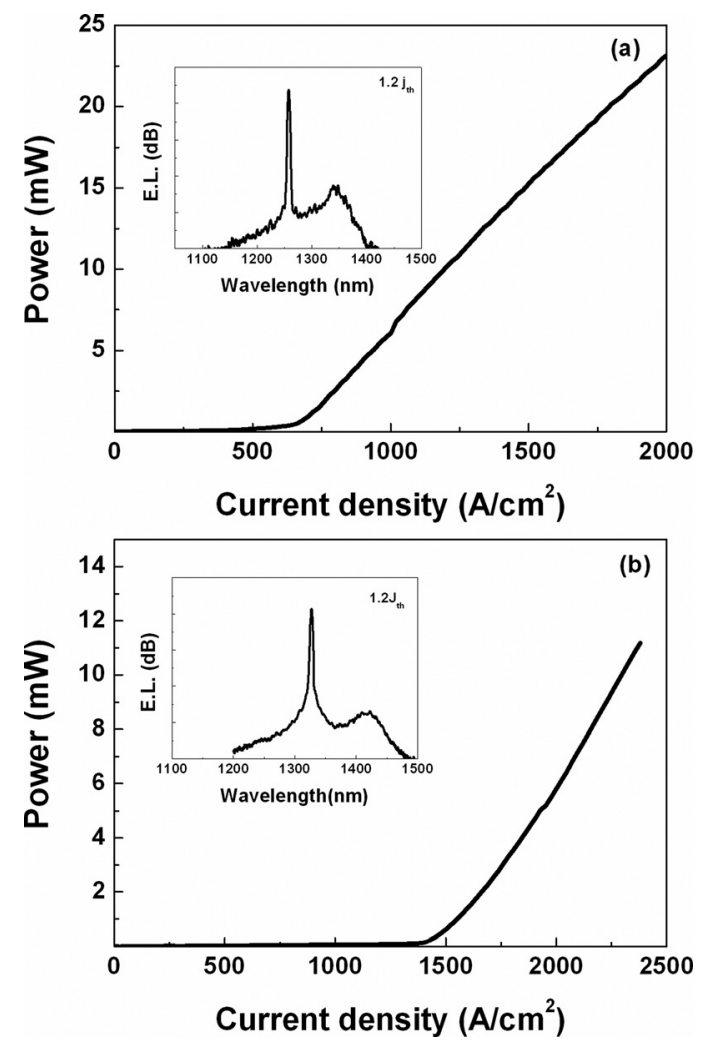

FIG. 2. (a) Total optical power (from both as-cleaved facets) as a function of current density from a $1 \mathrm{~mm}$-long broad area laser fabricated from GaAscapped bilayer sample. Inset: RT lasing spectrum obtained at a current injection of $1.2 \times \mathrm{J}_{\mathrm{th}}$. (b) Total optical power as a function of current density from a $6 \mathrm{~mm}$-long broad area laser fabricated from InGaAs-capped bilayer sample. Inset: RT lasing spectrum obtained at $1.2 \times \mathrm{J}_{\mathrm{th}}$.

not shown). The inset of Fig. 1(c) shows normalized photoluminescence (PL) spectra from GaAs- and InGaAs-capped bilayer test samples at RT. Emission at $1.34 \mu \mathrm{m}(1.42 \mu \mathrm{m})$ and $1.26 \mu \mathrm{m}(1.33 \mu \mathrm{m})$ for the GaAs-capped (InGaAscapped) samples is attributed to emission from the ensemble of ground states and excited states of the QDs, respectively. The linewidth of GS emission wavelength is only $15 \mathrm{meV}$ at RT for both the samples, which is comparable to the lowest PL linewidth reported for any QD ensemble. ${ }^{15}$ This is attractive for device applications, with low inhomogeneous broadening expected to benefits for the peak gain, threshold current, and modulation bandwidth of QD lasers. ${ }^{16}$

Fig. 2(a) shows typical optical power-current characteristics of a $1 \mathrm{~mm}$ long ridge laser device fabricated from the GaAs-capped bilayer sample, measuring the total output power from both as-cleaved facets using pulsed excitation with a pulse duration of $5 \mu \mathrm{s}$ and a $1 \%$ duty cycle to minimize heating. The laser exhibits ES lasing at $1.26 \mu \mathrm{m}$ with a threshold current density $\left(\mathrm{J}_{\mathrm{th}}\right)$ of $650 \mathrm{~A} \mathrm{~cm}^{-2}$ and a slope efficiency of $0.25 \mathrm{~W} / \mathrm{A}$. The inset shows the lasing spectra at 1.2 times $\mathrm{J}_{\text {th }}$. Fig. 2(b) shows power-current characteristics of a $6 \mathrm{~mm}$ long ridge laser device fabricated from the InGaAs-capped bilayer sample, using the same measurement conditions as for the GaAs-capped bilayer. The laser exhibits excited state lasing at $1.33 \mu \mathrm{m}$ but with a higher $\mathrm{J}_{\text {th }}$ of 1400 $\mathrm{A} \mathrm{cm}^{-2}$ and a reduced slope efficiency of $0.07 \mathrm{~W} / \mathrm{A}$. The lasing wavelengths for both the samples match the excited state EL peaks observed in Fig. 1 and are at a longer wavelength 


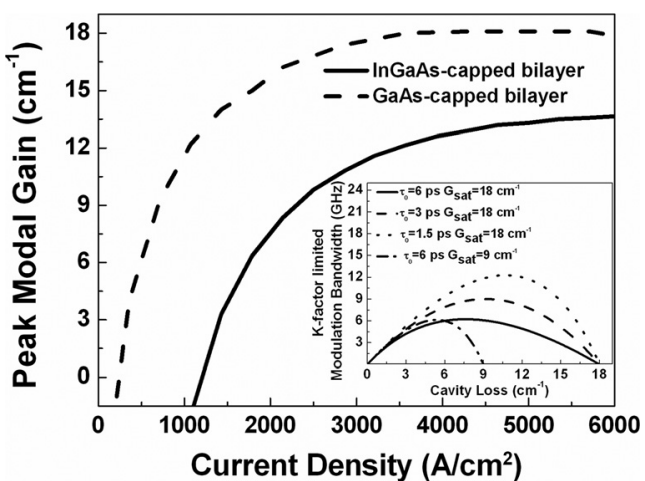

FIG. 3. Peak ES modal gain for GaAs-capped and InGaAs-capped bilayer structures obtained by multi-section gain measurements. Inset: K-factor limited modulation bandwidth as a function of cavity loss [after Ishida et al. (Ref. 5)] for fixed $\mathrm{G}_{\max }$ of 9 and $18 \mathrm{~cm}^{-1}$.

than the seed layer emission $(1.2 \mu \mathrm{m})$, indicating that lasing is occurring in the ES of the upper layer QDs. The increased device length required to achieve ES lasing (and corresponding increase in $\mathrm{J}_{\mathrm{th}}$ ) and the decrease in slope efficiency for InGaAs-capped bilayer laser is attributed to the increased optical losses in this sample.

Using multi-section devices, ${ }^{17}$ the gain spectrum may be determined as a function of current density. ${ }^{12}$ Absorption measurements and analysis of gain spectra at long wavelengths indicate an internal loss of $3 \pm 1 \mathrm{~cm}^{-1}$ for the GaAscapped and $5 \pm 1 \mathrm{~cm}^{-1}$ for the InGaAs-capped sample. These values for internal loss are combined with net modal gain values to plot the peak modal gain per dot of the ES for both samples as a function of current density in Fig. 3. ${ }^{18}$ This value is an important parameter in determining the damping limited modulation bandwidth. ${ }^{5,19}$ The saturated modal gain for the GaAs- and InGaAs-capped bilayer samples are determined to be 18 and $12 \mathrm{~cm}^{-1}$, respectively. The modal gain per QD of the GS and ES of the GaAs capped sample compares very favorably with commercially sourced QD laser samples operating at $1.28 \mu \mathrm{m}$, which have also been processed into multi-section devices. The commercially sourced material not only has four times the number of QDs in the active region (twice the number of QD layers, each with double the QD density in each layer) but also has around double the inhomogeneous line-width and exhibits an ES modal gain of $\sim 40 \mathrm{~cm}^{-1}$ at $1.2 \mu \mathrm{m}^{8}{ }^{8}$ This represents only $\sim 2$ times increase in modal gain compared to the bilayer QD lasers considered here. This difference is attributed to the smaller inhomogeneous linewidth for the bilayer QD devices. $^{20}$ An increase in the areal QD density in the bilayers, achievable by tuning the growth conditions of the seed layer, can be expected to further enhance the ES saturated gain. ${ }^{21}$

The inset to Fig. 3 shows the calculated K-factor limited modulation bandwidth as a function of scattering time to the lasing state, $\tau_{0}$, and the saturated gain of the lasing state, $G_{\text {sat }}$, after Ishida et $a l^{5,19}$ The scattering time to the GS has previously been determined to be $\sim 3$ ps (Refs. 5 and 7) for conventional 1.3 $\mu \mathrm{m}$ QD laser materials. A typical method to enhance the maximum modulation bandwidth is to include more QD layers within the active region. A calculated increase in the maximum K-factor limited bandwidth is observed for $\tau_{0}=3 \mathrm{ps}$ and increasing $\mathrm{G}_{\mathrm{sat}}$ from, e.g., $9 \mathrm{~cm}^{-1}$ to $18 \mathrm{~cm}^{-1}$. However, it has been shown that carrier transport effects become significant for large numbers of QD layers (i.e., thick active elements). ${ }^{22}$ The carrier transport time may be included as an effective increase in scattering time to the lasing state in the rate equation model $^{23}$ with carrier transport times rising from $\sim 1$ ps to $\sim 4 \mathrm{ps}$ for 5 and 10 QD layers, respectively. ${ }^{22}$ The impact of increased transport times on the maximum modulation bandwidth is observed by comparing the curve for $\mathrm{G}_{\mathrm{sat}}=18 \mathrm{~cm}^{-1}$ and $\tau_{0}=$ $6 \mathrm{ps}$ with that for the same saturated gain, $\mathrm{G}_{\mathrm{sat}}=18 \mathrm{~cm}^{-1}$, and the expected $\tau_{0}=3 \mathrm{ps}$. These cases illustrate the effect of doubling saturated gain at the expense of significant transport times, suggesting that essentially the same maximum modulation bandwidth would be obtained. Experimentally, a reduction in maximum modulation bandwidth has been observed for a 10 QD layer laser compared to a 5 QD layer laser due to this carrier transport effect. ${ }^{22}$ The advantage of an ES laser is that the net modal gain is increased without increasing the QD layer number. Indeed, the scattering time is expected to be reduced ${ }^{8}$ due to the higher density of final scattering states, leading to an increase in the predicted $\mathrm{K}$-factor limited bandwidth (see Fig. $\left.4, \mathrm{G}_{\mathrm{sat}}=18 \mathrm{~cm}^{-1}, \tau_{0}=3 \mathrm{ps}, \mathrm{G}_{\mathrm{sat}}=18 \mathrm{~cm}^{-1}, \tau_{0}=1.5 \mathrm{ps}\right)$.

In summary, excited state QD lasers spanning the O-band is made possible using either the GaAs or InGaAscapped capped bilayer structures. RT lasing from either the GS or ES is observed, depending on cavity length, for a range of wavelengths of 1.34-1.42 $\mu \mathrm{m}$ from the GS or 1.26$1.33 \mu \mathrm{m}$ for the ES. The saturated peak modal gain per QD from these devices is comparable to commercially available $1.3 \mu \mathrm{m}$ QD lasers operating in the GS, but the reduced number of QD layers required for ES lasing and in the increased degeneracy of states is expected to lead to an increase in the $\mathrm{K}$-factor limited bandwidth for these devices.

${ }^{1}$ D. L. Huffaker et al., Appl. Phys. Lett. 73, 2564 (1998).

${ }^{2}$ K. Mukai and M. Sugawara, Appl. Phys. Lett. 74, 3963 (1999).

${ }^{3}$ Y. Tanaka et al., in Proceedings of optical fibre communication, IEEE, San Diego, USA (2009).

${ }^{4}$ S. Fathpour et al., J. Phys. D: Appl. Phys. 38, 2103 (2005).

${ }^{5}$ M. Ishida et al., Appl. Phys. Lett. 85, 4145 (2004).

${ }^{6}$ A. Salhi et al., IEEE J. Sel. Top. Quantum Electron. 14, 1188 (2008).

${ }^{7}$ R. R. Alexander et al., IEEE J. Quantum Electron. 43, 1129 (2007).

${ }^{8}$ B. J. Stevens et al., Appl. Phys. Lett. 95, 061101 (2009).

${ }^{9}$ C. S. Lee et al., Appl. Phys. Lett. 98, 011103 (2011).

${ }^{10}$ E. C. Le Ru et al., Phys. Rev. B 67, 165303 (2003).

${ }^{11}$ E. Clarke et al., J. Appl. Phys. 107, 113502 (2010).

${ }^{12}$ P. Blood et al., IEEE J. Sel. Top. Quantum Electron. 9, 1275 (2003).

${ }^{13}$ Q. Xie et al., Phys. Rev. Lett. 75, 2542 (1995).

${ }^{14}$ W. M. McGee et al., J. Appl. Phys. 99, 043505 (2006).

${ }^{15}$ Z. Mi and P. Bhattacharya, J. Appl. Phys. 98, 023510 (2005).

${ }^{16}$ L. V. Asryan and R. A. Suris, Appl. Phys. Lett. 96, 221112 (2010).

${ }^{17}$ P. D. L Judson et al., IET Optoelectron. 3, 100 (2009).

${ }^{18}$ M. A. Majid et al., IEEE J. Sel. Topics Quantum Electron. (2011), in press.

${ }^{19}$ M. Ishida et al., J. Appl. Phys. 101, 013108 (2007).

${ }^{20}$ M. Sugawara et al., Phys. Rev. B 61, 7595 (2000).

${ }^{21}$ C. Y. Ngo et al., Appl. Phys. Lett. 95, 181913 (2009).

${ }^{22} \mathrm{M}$. Ishida et al., in $22^{\text {nd }}$ IEEE, International semiconductor laser conference (2010)

${ }^{23}$ R. Nagarajan et al., IEEE Photon. Technol. Lett. 4, 121 (1992). 